\title{
Comprensión lectora en los jóvenes del Bachillerato La Salle Ayahualulco
}

\author{
De la Torre Juárez Alexis Manuel ${ }^{1}$ \\ Hernández Gutiérrez Clara ${ }^{2}$ \\ Monroy García Mariela ${ }^{3}$
}
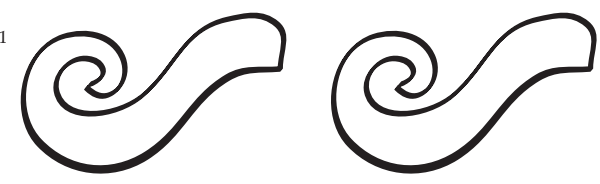

\section{Resumen}

La siguiente investigación tiene el objetivo de conocer más acerca de los problemas de la comprensión lectora que se tienen en la comunidad de Ayahualulco, Veracruz; el trabajo se realizó en el semestre agosto - diciembre, 2012.

Se cumplió un proceso metodológico cualitativo, se conoció previamente el contexto cultural y social de la entidad sujeta a investigación, indagando en libros y portales electrónicos. Se procesaron y aplicaron instrumentos en dicha comunidad y sus alrededores para así conocer a fondo el problema lector que rodea a los jóvenes de la comunidad y obtener datos confiables y exactos que fueron validados y triangulados para así llegar a las conclusiones.

\section{Introducción}

\section{I.1 Planteamiento del problema}

Según datos del Instituto Nacional para la Evaluación de la Educación a primaria sólo ingresan en su edad normativa y adelantados 2,291,521 niños de 6 años, lo cual representa el 99.3\% de los 2,305,431 niños registrados con 6 años de edad (Mexicanos Primero, 2011). Se debe recordar que las bases del aprendizaje de la comprensión lectora se construyen durante la primaria.
En la actualidad, muchos niños y jóvenes aprenden a leer pero no a entender el mensaje que la lectura pretende transmitir. Cuando no se adquieren las habilidades de la comprensión lectora, se torna complicado poder distinguir ideas principales, desarrollar el gusto por la lectura, así como captar el mensaje del texto.

El problema central a atender en este trabajo es: el desarrollo de la comprensión lectora en los jóvenes que estudian en el Bachillerato La Salle Ayahualulco.en el periodo de agostodiciembre de 2012.

\section{I.1.1 Objetivos}

Objetivo General:

- Indagar el nivel de comprensión lectora que poseen los jóvenes del Bachillerato La Salle Ayahualulco.

Objetivos Específicos:

- Averiguar si los jóvenes del Bachillerato La Salle de Ayahualulco logran extraer el significado de un texto.

- Identificar si los jóvenes del Bachillerato La Salle son capaces de implementar estrategias para la comprensión de textos.

- Identificar los factores que afectan el desarrollo de la comprensión lectora. 




I.1.2 Preguntas de Investigación

1. ¿Los jóvenes bachilleres Lasallistas son capaces de comprender el sentido de un texto?

2. ¿Los jóvenes del Bachillerato La Salle Ayahualulco son capaces de implementar estrategias para la comprensión de textos?

3. ¿Qué importancia tiene que los jóvenes del Bachillerato La Salle Ayahualulco comprendan un texto?

4. ¿Qué factores afectan el desarrollo de la comprensión lectora en los jóvenes del Bachillerato La Salle Ayahualulco?

\section{I.1.3 Justificación}

La presente investigación está basada en las urgencias propuestas en el Proyecto Educativo Regional Lasallista Latinoamericano (PERLA) y se basa principalmente en la urgencia de Democratización del conocimiento, que tiene como principal objetivo orientar a la innovación y a la transformación social y a las necesidades de los más desfavorecidos y del contexto para así asegurar el acceso a la educación.

\section{Marco Teórico}

\section{II.1 Marco Histórico}

En México, se han implementado varios programas para fortalecer la lectura, la comprensión lectora y la escritura. Uno de ellos proyecto PALE (Propuesta para el Aprendizaje de la Lengua Escrita), desarrollado por Margarita Gómez Palacio en 1981 (Ferreiro, 2002). Dicha propuesta fue diseñada para atender a niños que no habían aprendido a leer y escribir.

Otro proyecto es el PRONALEES (Programa Nacional para el Fortalecimiento de la Lectura y la Escritura en Educación Básica) que inició en 1995(Secretaría de Educación de Jalisco, $\mathrm{s} / \mathrm{f}$ ), con el fin de que los alumnos lograran adquirir y consolidar la capacidad de leer y expresarse de manera oral y escrita.



Actualmente el Programa Nacional de Lectura (PNL) "propone mejorar las competencias comunicativas en los estudiantes de educación básica y favorecer el cambio escolar a través de una política de intervención que asegura la presencia de materiales de lectura que apoyen el desarrollo de hábitos lectores y escritores de alumnos y maestros" (SEP, 2012).

Se puede apreciar que, durante décadas en México, se ha buscado reforzar la comprensión lectora. Prueba de ello son las ferias de libro que se han organizado con el fin de que la población encuentre el gusto por la lectura. Así como la campaña Diviértete leyendo emprendida por el Consejo de la Comunicación: voz de las empresas, realizada con intelectuales y artistas que promueven veinte minutos de lectura con los niños, con el objetivo de fomentar la lectura.

\section{II.2 Marco Conceptual}

Comprensión lectora: Es una reinterpretación significativa y personal de los símbolos verbales impresos, que se justifica solo en la medida en que el lector es capaz de comprender los significados que están a su disposición (González, 2007).

Comprensión lectora: Forma en que el sujeto se relaciona con la lengua escrita. Implica que el sujeto esté familiarizado con los grafonemas, los signos de puntuación y otros signos auxiliares como los asteriscos, los guiones o los paréntesis y su aporte a la claridad de la información. Es también, la apropiación de los significados de un texto mediante el uso del contexto, la predicción, la recapitulación y la jerarquización de la información (González, 2007).

Hábito: Costumbre o páactica adquirida por la frecuencia de repetición de un acto (Diccionario de las Ciencias de la Educación, 2003).

Lectura: Es el proceso de significación y comprensión de algún tipo de información o ideas almacenadas en un soporte y transmitidas mediante algún tipo de código, usualmente lenguaje (Diccionario de las Ciencias de la Educación, 2003). 


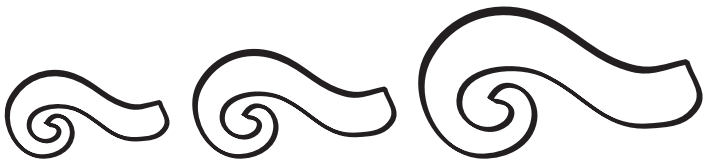

Para efecto de esta investigación, comprensión lectora se define como: una reinterpretación personal y significativa del mensaje transmitido por un texto, que se justifica solo en la medida en que el lector es capaz de comprender los significados que están a su disposición.

\section{II.3 Marco Teórico}

Aprender a leer, significa poder tener el acceso a la cultura y al conocimiento en la sociedad. La importancia de la alfabetización cada vez va en aumento, la sociedad actualmente se encuentra más preocupada, con la constante extensión y diversificación de los usos de texto escritos que posibilita la integración de las tecnologías en educación.

Es por eso que la comprensión lectora, es un tema de interés social permanente y no exclusivamente de los ámbitos escolares. La enseñanza lectora es contemplada como una tarea propia de los profesores de todas las áreas de conocimiento, pero en realidad una parte importante del aprendizaje escolar, consiste en la ampliación en el ámbito lingüístico.

Según Colmer (1993), la enseñanza de la lectura ha implementado un movimiento de integración y articulación entre los aprendizajes sobre la forma del escrito y sobre su función, entre las habilidades que se ponen en juego y el sentido que la lectura tiene para los lectores. Enseñar a entender un texto, se ha convertido en el objetivo principal en las prácticas escolares y esto ha permitido experimentar y articular con nuevas prácticas para obtenerlo.

Un nuevo modelo de lectura establecido por la investigación supone la interrelación de tres factores (Irwin, 1986 en Colomer, 1993) que deben de tomarse en cuenta en la programación de la enseñanza: el lector, el texto y el contexto de la lectura.

1. El lector incluye los conocimientos que éste posee en un sentido amplio, es

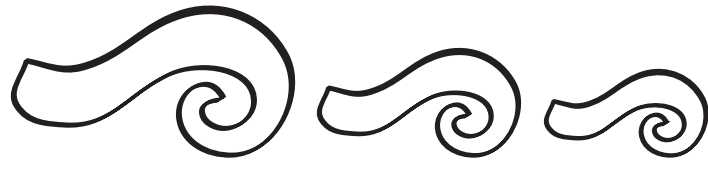

decir, todo lo que es y sabe sobre el mundo, así como todo lo que hace durante la lectura para entender el texto.

2. El texto se refiere a la intención del autor, a contenido de lo que dice y a la forma que ha organizado su mensaje.

3. El contexto comprende las condiciones de la lectura, tanto las que se fija el propio lector como las derivadas del entorno social.

La relación entre estas tres variables, influye en la posibilidad de que la comprensión del texto y las actividades escolares cuenten con una organización lógica, y así poder hacer que los interesados en la lectura tengan un aprendizaje significativo.

La Secretaría de Educación Pública (s/f), mediante el Manual de procedimientos para el fomento y la valoración de la competencia lectora en el aula, expresa la importancia de la lectura y se cita a continuación:

La lectura es un proceso complejo que cada persona realiza por sí misma; éste le permite examinar el contenido de lo que lee, analizar cada una de sus partes para destacar lo esencial y comparar conocimientos ya existentes con los recién adquiridos.

La lectura es importante porque:

Potencia la capacidad de observación, de atención y de concentración.

Ayuda al desarrollo y perfeccionamiento del lenguaje. Mejora la expresión oral y escrita y hace el lenguaje más fluido. Aumenta el vocabulario y mejora la ortografía.

Facilita la capacidad de exponer los pensamientos propios.

Amplía los horizontes del individuo permitiéndole ponerse en contacto con lugares, gentes y costumbres lejanas a él en el tiempo o en el espacio.

Estimula y satisface la curiosidad intelectual y científica. 


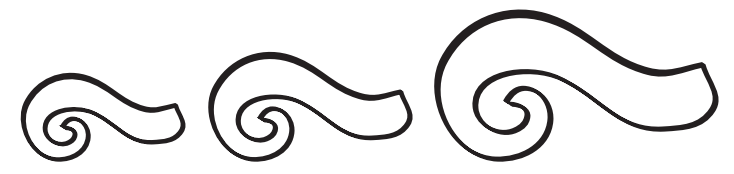

Desarrolla la capacidad de juicio, de análisis, de espíritu crítico. El niño lector pronto empieza a plantearse porqués.

Es una afición para cultivar en el tiempo libre, un pasatiempo para toda la vida.

\section{II.4 Marco Referencial}

Asimismo se revisó la investigación Diagnóstico de comprensión lectora en educación básica en Villarrica y Loncoche, Chile elaborada por Liliana Inés Fuentes Monsáleves.

Los resultados de la muestra total se presentan de acuerdo al nivel de procesamiento, tipo de texto y habilidades evaluados.

"Es importante mencionar que los porcentajes de los resultados corresponden en sí mismos a un 100 por ciento y no al total de la prueba, por lo que los valores no deben completar un 100 por ciento"(Fuentes, 2009).

Los niveles de procesamiento alcanzados en la muestra total son: $48 \%$ el nivel superficial, $35 \%$ el nivel medio y un $33 \%$ el nivel profundo.

El porcentaje de comprensión alcanzado por la muestra total, en los dos tipos de texto evaluados es: en el texto narrativo un $41 \%$ y en el texto expositivo un $36 \%$ (Fuentes, 2009).

Por otro lado la investigación titulada "El nivel de comprensión lectora en sexto grado de primaria" elaborada por María Elena Velásquez Pérez en el año 2006, señala a partir de los resultados arrojados por la aplicación del instrumento, se encuentra que 62 alumnos son ubicados en el nivel 1 (literal); 8 estudiantes en el segundo que es el de reorganización; en el tercero que es el inferencial o interpretativo y el cuarto correspondiente al nivel crítico o de juicio, no hay alumnos.

Asimismo se analiza que el promedio de los alumnos con el $88.6 \%$ en comprensión lectora

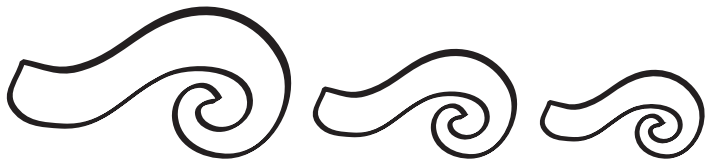

se sitúa dentro del componente literal; al contar con los elementos básicos para repetir la información, tal y como se maneja en el texto; limitándose solamente a lo que la página impresa señala sin llegar a hacer una interacción significativa con el escrito. Aprenden a codificar pero no logran comprender; solamente son capaces de realizar las tareas más elementales de lectura al identificar la información específica de un texto.

También haciendo referencia al nivel de comprensión lectora inferencial o interpretativo cabe señalar que ninguno de los educandos alcanzo este, el cual se distingue porque los lectores pueden explicar, comentar o generalizar la información contenida en un escrito a situaciones de la vida cotidiana.

\section{Método}

\section{I.1 Enfoque}

La presente investigación siguió el enfoque cualitativo, caracterizado por capturar una realidad dinámica, pues tendrá capacidad de irse transformando, según se desarrolle la misma. La realidad estudiada fueron las condiciones en que se encontraba la comprensión lectora de los jóvenes del Bachillerato La Salle Ayahualulco.

Muestra una perspectiva interna, pues se analizará la parte subjetiva de la comprensión lectora de los jóvenes de Ayahualulco.

El enfoque de la investigación es holístico, se analizarán todos los aspectos involucrados con el objeto de estudio, es decir la comprensión lectora.

El diseño de la investigación estará orientado al proceso, pues se construirá a través de los hechos investigados en la comunidad.

Su estructura está abierta a los hechos, por lo cual pretende adaptarse a los sucesos y a la 




información obtenida en el proceso de investigación, entendiendo por información obtenida, los niveles de comprensión lectora con los que los jóvenes del Bachillerato La Salle Ayahualulco cuentan, así como los factores que influyen para el desarrollo de la misma.

El procedimiento que se llevará a cabo, es flexible, pues puede adaptarse a las condiciones en las que se encuentren los jóvenes de Ayahualulco.

Las condiciones en las que se desarrollará la investigación, son naturales. Es decir, se asistirá a la población de Ayahualulco para hacer trabajo de campo, alterando lo menos posible el contexto.

Los datos que se obtengan serán subjetivosobjetivos, se pretenden obtener mediante entrevistas, historias de vida y entrevistas. Y su análisis de llevará a cabo en la fase número tres del desarrollo de la investigación cualitativa, la fase analítica. En primera instancia se reducirán los datos que se obtengan, posteriormente se codificarán y al final se generarán resultados y conclusiones.

\section{2 Contexto}

A continuación se cita la información obtenida del portal de internet Veracruz.com (s/f):

Ayahualulco se localiza en la zona centro del estado de Veracruz. Limita al norte con Perote, al noreste con Xico, al este con Ixhuacán de los Reyes y al sur con el Estado de Puebla. Su distancia aproximada por carretera a la capital del estado es de 80 $\mathrm{Km}$. Su superficie es de $148.06 \mathrm{Km} 2$, cifra que representa un $0.20 \%$ del total del Estado. Tiene una población 23,431 habitantes.

Su nombre significa "Lago redondo"; del náhuatl Atl; Agua, Yahualli; corona o cosa redonda y Co; partícula locativa. Su historia lo señala como uno de los últimos pueblos totonacas de la vertiente del Golfo.

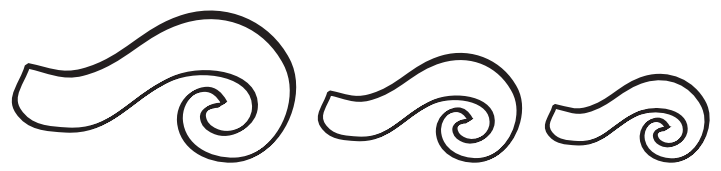

Posee un clima templado-extremoso, su temperatura media anual es de $10^{\circ} \mathrm{c}$; con lluvias abundantes en el verano y a principios del otoño, con menor intensidad en invierno. Su precipitación media anual es de 463 milímetros.

Entre sus atractivos culturales y turísticos destaca el monumento histórico El Fortín, ubicado en la avenida Niños Héroes, en la cabecera municipal. Entre sus fiestas y tradiciones se celebran el 24 de junio se la fiesta patronal de San Juan Bautista en Los Altos; el 25 de julio se festeja la fiesta en honor de Santiago Apostol en la cabecera municipal; del 24 al 27 de julio se celebra la fiesta religiosa en honor del señor Santiago con bailables populares, danzas autóctonas, carreras de caballos, actos religiosos, pelea de gallos, jaripeos y cabalgatas. Sus danzas típicas son Los Moros y Los Toriteros.

Realizan artesanías con fibras naturales, se dedican a la elaboración de escobas y escobetillas, así como a la creación de muebles rústicos de madera. De su gastronomía son tradicionales la barbacoa blanca y roja. Además, Ayahualulco cuenta con 2,000 hectáreas para ciclismo a campo traviesa en el poblado de Aguazuelas.

\section{III.4 Diseño}

El diseño con el cual está determinada esta investigación es de tipo transversal ya que sólo será estudiada por nuestra parte en la comunidad de Ayahualulco por un tiempo determinado y ya que no es posible seguir dándole seguimiento. Sin embargo, con el trabajo que se realizará en esta investigación propondremos estrategias para que puedan seguir siendo usadas a largo plazo en los niños de la comunidad de Ayahualulco.

\section{III.5 Sujeto, universo y muestra}

Con base en los instrumentos aplicados en la comunidad de Ayahualulco a continuación se pretende describir la población de la cual se obtuvo la información de las encuestas, guías de observación e historias de vida. 


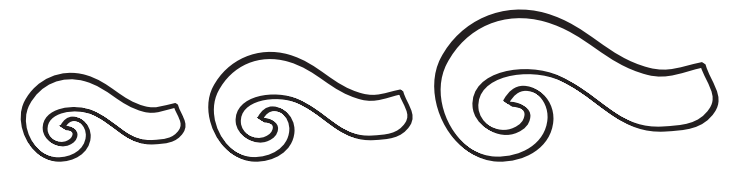

Para la aplicación de las entrevistas, se realizó un visiteo en las diferentes rancherías de la comunidad de Ayahualulco las cuales fueron: Coscomatepec, Chirinos, La manzanita, La Mesa, Rancho Alegre, Benito Juárez, y Colcalcingo, Yolotla, con la finalidad de platicar un poco con la gente y conseguir la información acerca de la lectura de comprensión que llevan a cabo los padres con los hijos. En la tabla posterior se muestra el número de entrevistas aplicadas por rancherías.

Los sujetos de las rancherías en su mayoría son personas alegres, amables y cordiales ya que a pesar de las condiciones en las que se encuentran fueron muy accesibles a la hora de platicar con nosotros y a su vez algunos de ellos nos ofrecían comida.

También se realizó un recorrido por las diferentes escuelas que se encontraban en los ranchos, los maestros se encontraban trabajando con un gran número de alumnos, no obstante, todos estaban dispuestos a responder la entrevista.

El número de entrevistas aplicadas a padres fue de 31 y de entrevistas aplicadas a maestros fue de 9, sumando un total de 40 entrevistas aplicadas en las diferentes rancherías: (Tabla 1)

\begin{tabular}{|l|c|c|}
\hline Rancherías & Entrevistas a Padres & Entrevistas a Maestros \\
\hline Coscomatepec & 8 & \\
\hline Chirinos & 6 & 2 \\
\hline La Manzanita & 3 & 2 \\
\hline Ayahualulco & 6 & 4 \\
\hline La mesa & 4 & \\
\hline Rancho alegre & 3 & \\
\hline Benito Juárez & 1 & \\
\hline Colcalcingo, Yolotla & & 1 \\
\hline TOTALES & 31 & 9 \\
\hline
\end{tabular}

Para continuar con la investigación se aplicaron 30 historias de vida y 30 guías de observación a un grupo de bachillerato con un número de 57 alumnos de La Salle Ayahualulco, los alumnos iban desde los 17 años de edad hasta los 18 años. Por otro lado los jóvenes siempre mostraron interés por los talleres impartidos y su actitud fue muy positiva a la hora de responder las historias de vida.

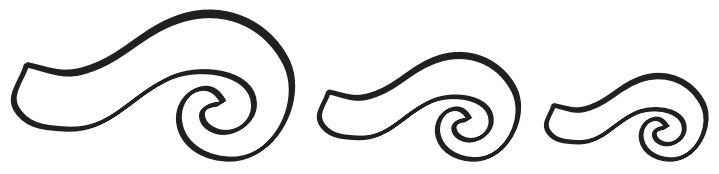

La muestra total de las historias de vida fue de 30, aplicadas a un grupo de 57 alumnos, sin embargo 2 de las historias de vida fueron invalidadas por omisión. Por último se realizó una observación con la ayuda de un instrumento realizado previamente al mismo grupo que participó en la aplicación de las historias de vida.

\section{III.7 Análisis de datos}

\section{Análisis de entrevistas}

Según las entrevistas realizadas tanto a padres y maestros para esta investigación en la comunidad de Ayahualulco los resultados que arrojan después del análisis de cada una de éstas es el siguiente:

En cuanto a qué tan importante es la lectura para los padres de familia en la comunidad, el 97\% de los encuestados considera que es importante (gráfica 1), en el caso de los maestros el $100 \%$ contesto positivamente (gráfica 2).

\section{Gráfica 1}



Gráfica 2

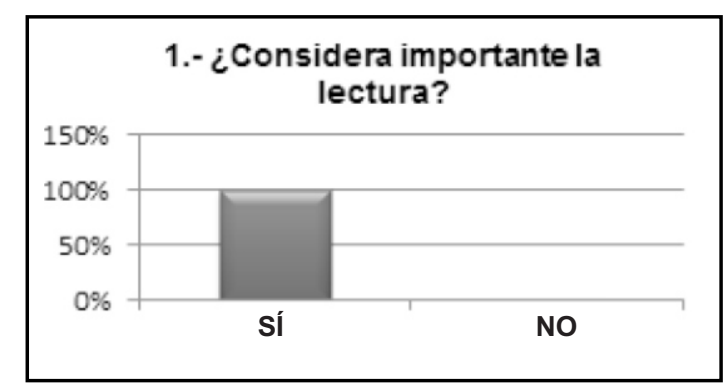




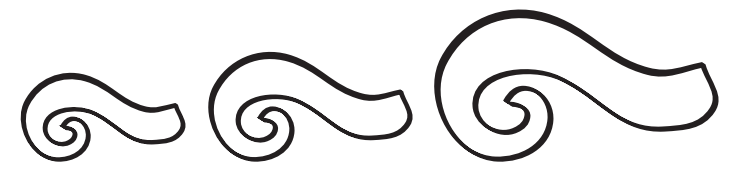

En la comunidad de Ayahualulco el $77 \%$ de las personas a las que se les realizó la encuesta fomentan el hábito de la lectura en su hogar (gráfica 1.1), en tanto a los maestros el 100\% lo hace en sus clases cada día (gráfica 2.1).

\section{Gráfica 1.1}

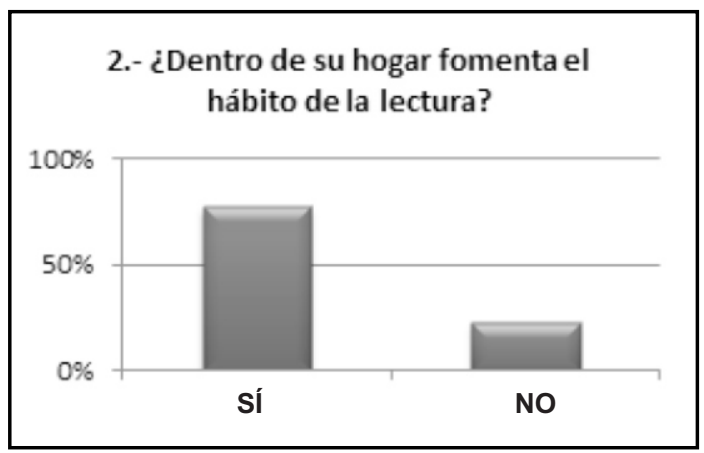

\section{Gráfica 2.1}

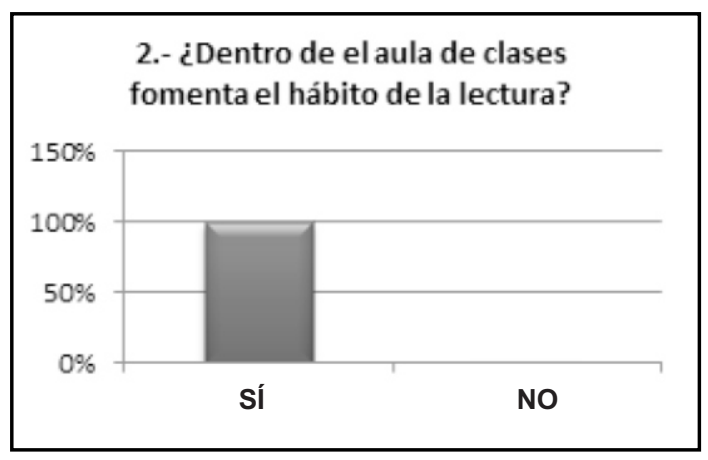

Con relación a la frecuencia con la que los padres se dan cuenta de que sus hijos toman libros para leer, el $42 \%$ de los padres nota que solo en algunas veces sucede eso, seguido del $39 \%$ que lo hace frecuentemente y un 19\% nunca lo hace (gráfica 1.3), en cuanto a los maestros encuestados en la comunidad el $100 \%$ de ellos menciona que sus alumnos toman frecuentemente un libro para leerlo en clase (gráfica 2.3).

\section{Gráfica 1.3}

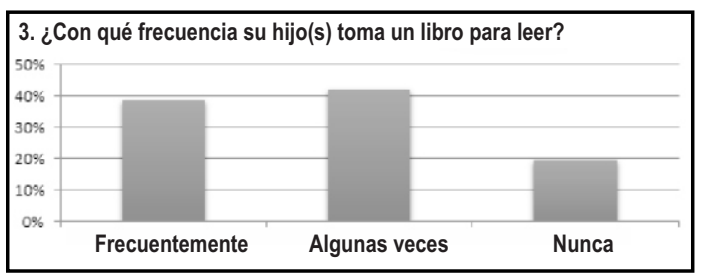

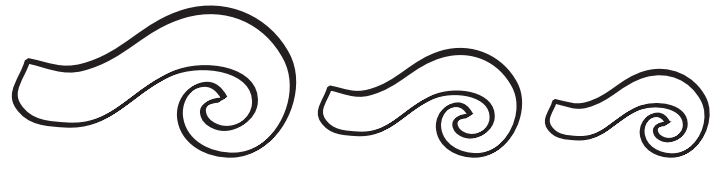

Gráfica 2.3

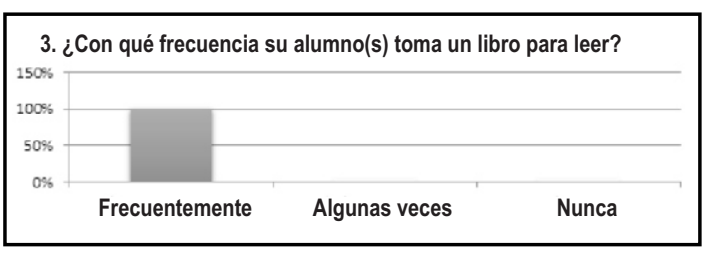

El $50 \%$ de los padres contestaron que la única razón por la que leen con sus hijos es por motivos escolares, el 25\% lo hace por gusto, el $3 \%$ lo hace por obligación y lamentablemente el 22\% nunca lee con sus hijos (gráfica 1.4), y de los nueve maestros encuestados el $60 \%$ de ellos realizan lecturas con sus alumnos por motivos escolares y el $40 \%$ por gusto (gráfica 2.4).

\section{Gráfica 1.4}

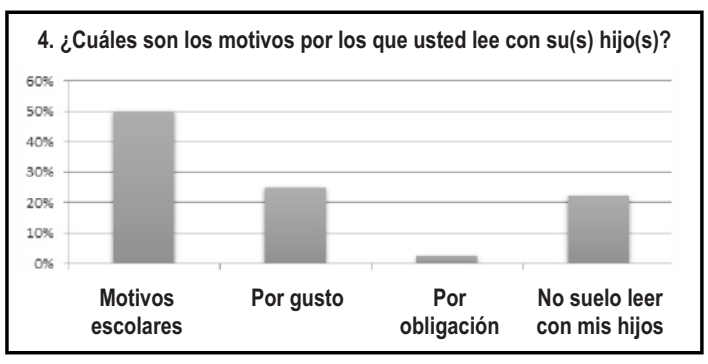

\section{Gráfica 2.4}

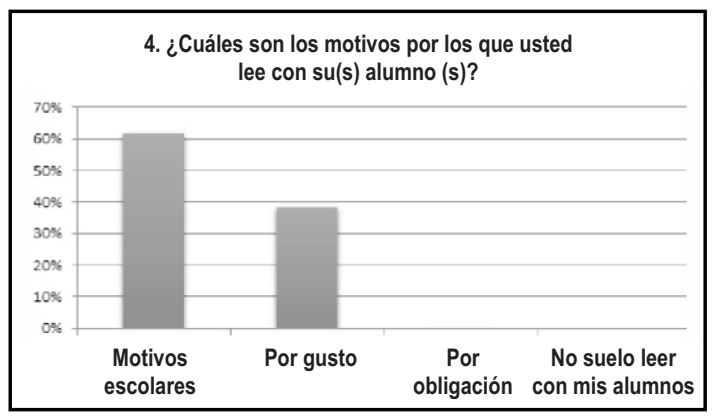

Al 47\% de los padres encuestados los temas que les resultan más interesantes para leer son los textos escolares, ya que es en el momento donde se toman un poco de tiempo para leer con ellos, el 16\% leen textos de entretenimiento, el $13 \%$ textos periodísticos y el $16 \%$ no suelen leer con sus hijos (gráfica 1.5). En el ámbito escolar el $70 \%$ de los maestros mencionaron 


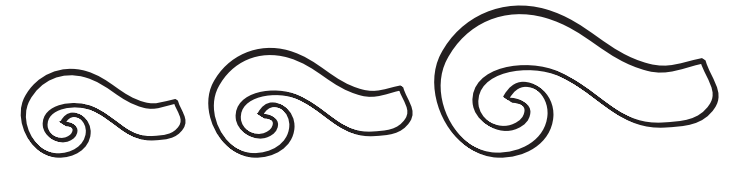

que lo que más les gusta leer con sus alumnos son textos literarios y el $30 \%$ prefieren textos de entretenimiento (gráfica 2.5).

\section{Gráfica 1.5}

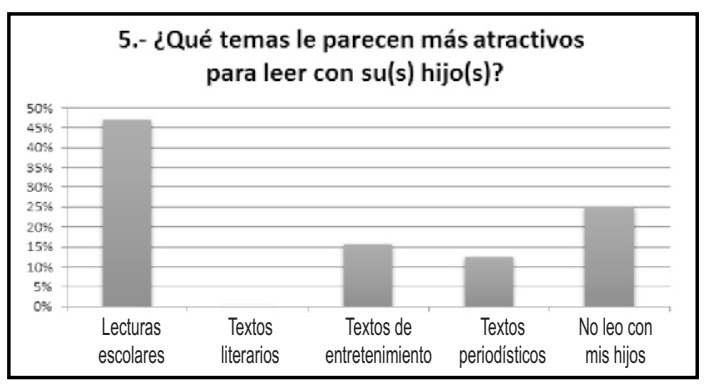

\section{Gráfica 2.5}

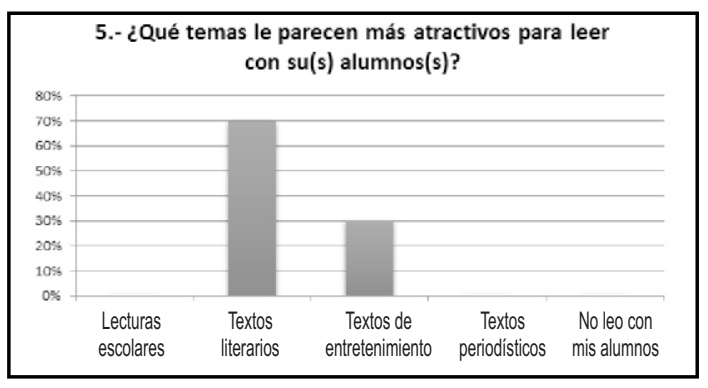

Con relación a la comprensión, el $84 \%$ de los padres se dan cuenta de que sus hijos comprendieron la lectura realizada y el $16 \%$ notan que no lograron hacerlo (gráfica 1.6), y el 90\% de los profesores notan que sus alumnos sí comprenden lo que leen y el $10 \%$ no pueden observarlo (gráfica 2.6)

\section{Gráfica 1.6}

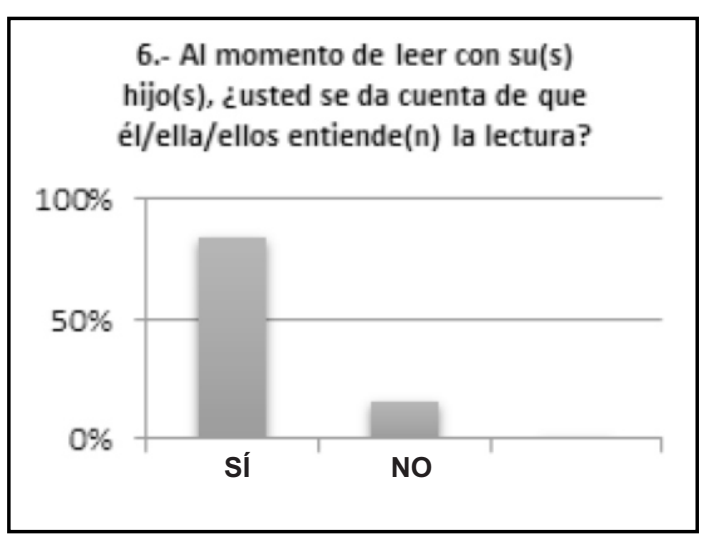

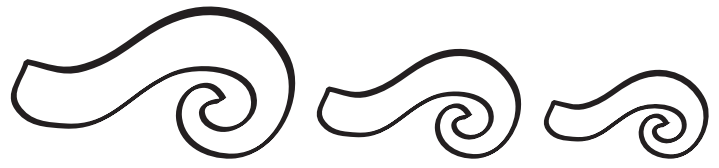

\section{Gráfica 2.6}

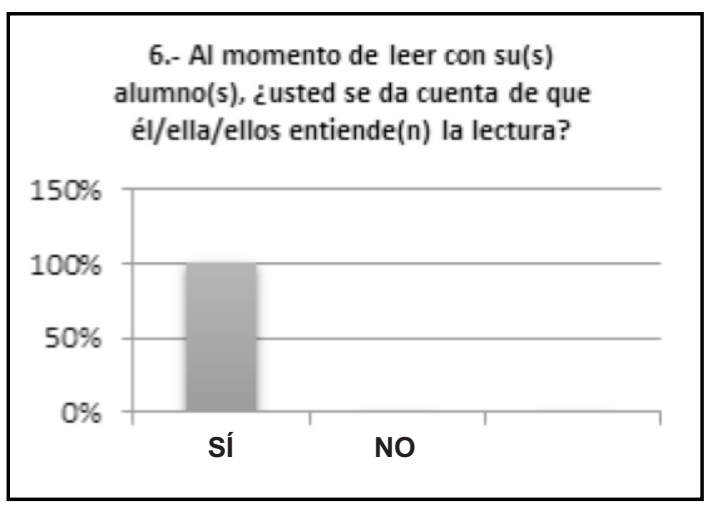

En la mayoría de las encuestas aplicadas con relación al aprendizaje que les dejan las lecturas al final de realizarlas, el $81 \%$ de los padres de familia notan que sí les dejan un aprendizaje significativo mientras que $19 \%$ no logra apreciarlos (gráfica 1.7), los maestros, por su parte, notan que al $90 \%$ de sus alumnos les deja un aprendizaje significativo que lo lleven a la práctica y en un $10 \%$ de sus alumnos no se aprecia (gráfica 2.7).

\section{Gráfica 1.7}

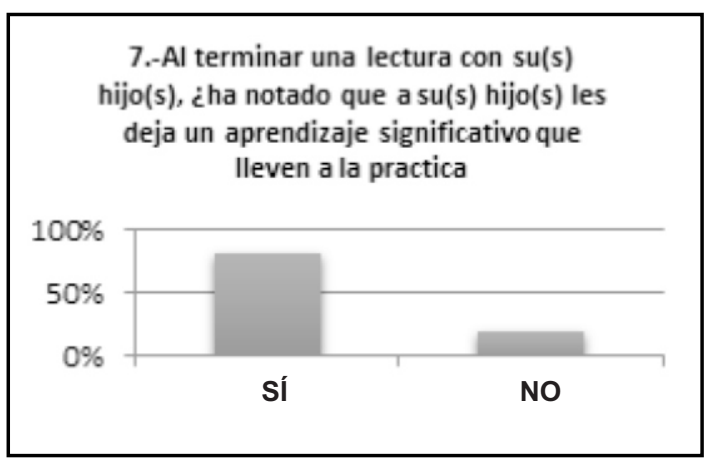

\section{Gráfica 2.7}

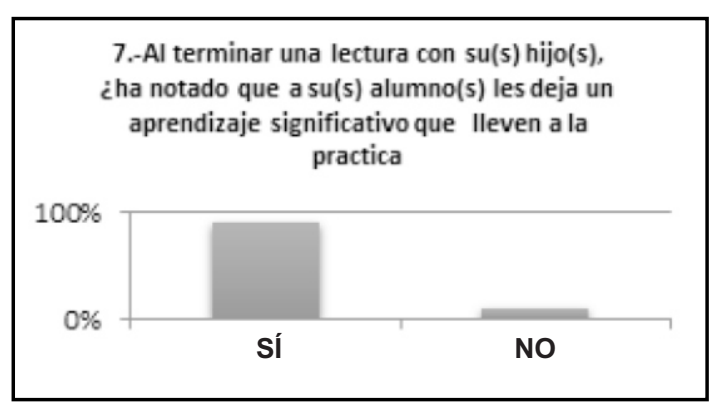




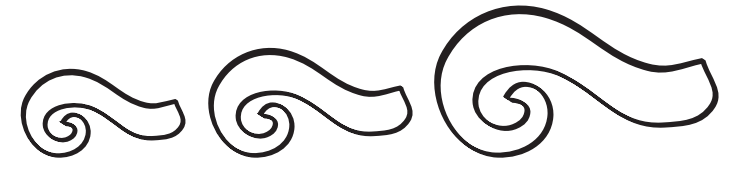

El tiempo que dedican los padres de familia a la lectura con sus hijos en la comunidad de Ayahualulco conforme a la encuestas aplicadas el $58 \%$ menciona que realizan más de 20 minutos de lectura, el 19\% no realiza ningún tipo de lectura, el 13\% únicamente lee de 10 a 20 minutos y el 10\% solamente lee de 5 a 10 minutos (gráfica 1.8), en cuanto a los maestros encuestados el $89 \%$ de ellos realizan más de 20 minutos de lectura con sus alumnos y el 11\% únicamente realiza de 10 a 20 minutos (gráfica 2.8).

\section{Gráfica 1.8}

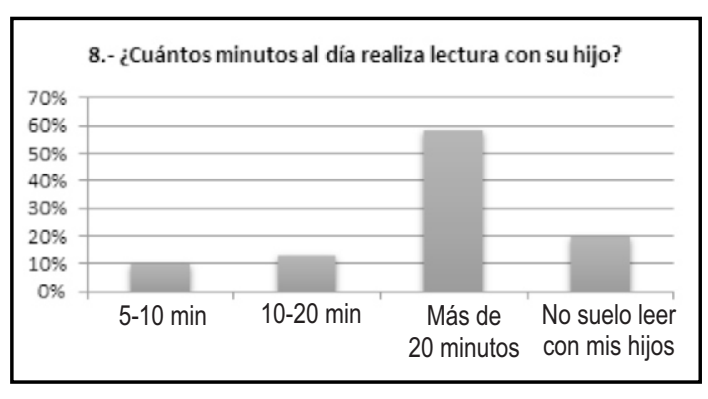

\section{Gráfica 2.8}

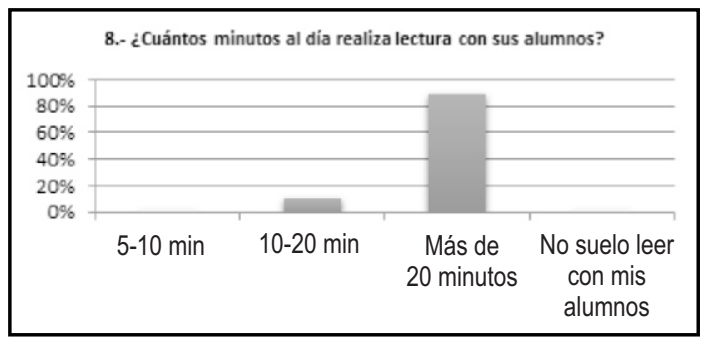

La manera en que los padres encuestados se dan cuenta que sus hijos han comprendido la lectura el $47 \%$ de ellos menciona que comentan la lectura, el $41 \%$ lo hace mediante preguntas y el $12 \%$ no lee con sus hijos (gráfica 1.9), el $89 \%$ de los maestros dijeron que se dan cuenta que han comprendido la lectura por medio de la realización de preguntas, mientras que el $14 \%$ sólo comentan la lectura (gráfica 2.9).

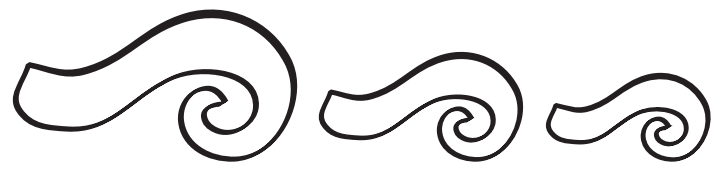

\section{Gráfica 1.9}

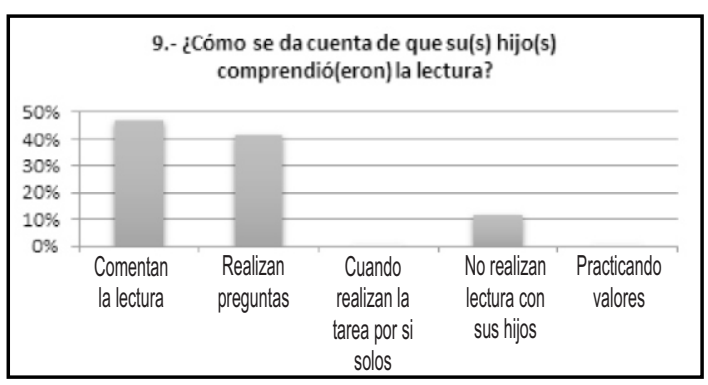

Gráfica 2.9

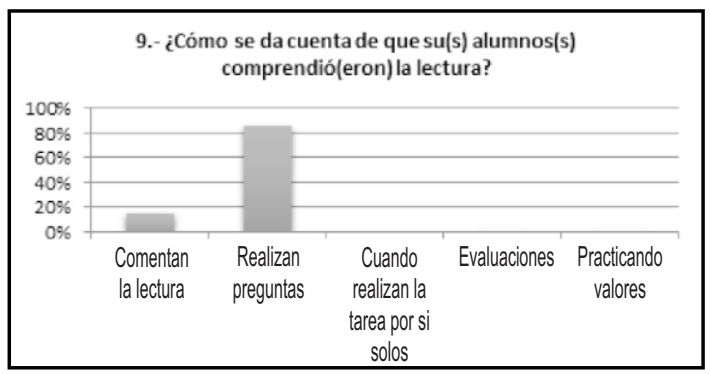

En la mayoría de las encuestas de los padres de la comunidad el $42 \%$ menciona que slo en algunas ocasiones realizan comentarios finales de lectura, mientras que el $26 \%$ lo hace siempre, el 19\% nunca realiza ningún tipo de comentarios y el $13 \%$ casi siempre lo hace (gráfica 1.10), con relación a los maestros el $67 \%$ mencionó que siempre realizan comentarios al final de cada una de las lecturas, el $11 \%$ casi siempre lo hace, el $11 \%$ solamente lo hace algunas veces y el $11 \%$ restante nunca lo hace (gráfica 2.10).

\section{Gráfica 1.10}

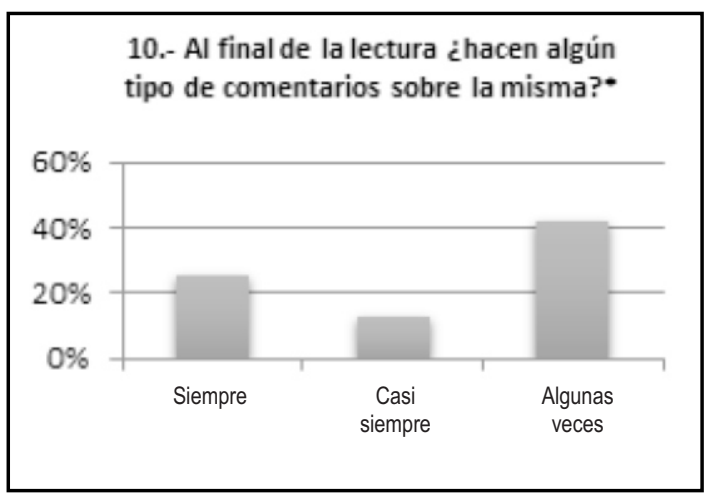




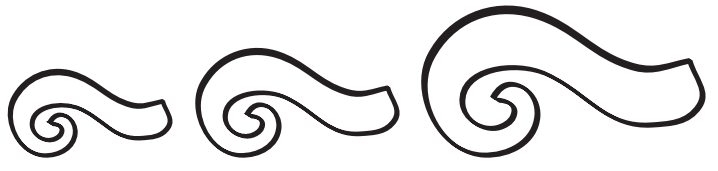

Gráfica 2.10

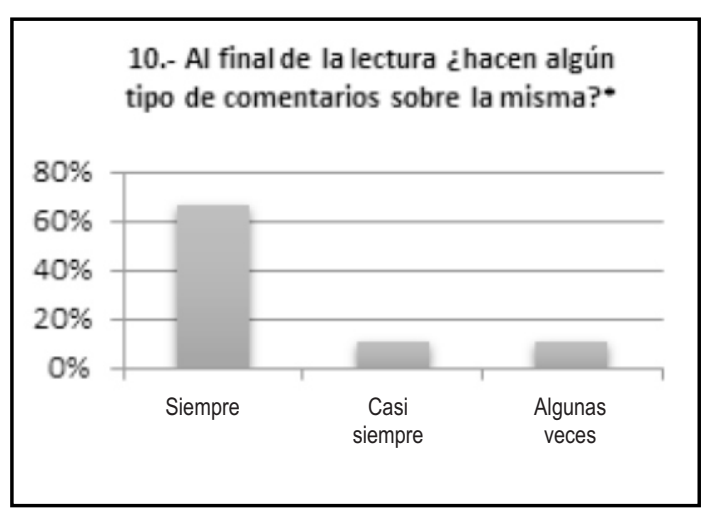

Análisis de observaciones

Los aspectos que se observaron en 30 jóvenes de 5to semestre del Bachillerato La Salle fueron:

1. Lee las instrucciones antes de comenzar la actividad

2. Se concentra al momento de leer

3. Subraya ideas principales

4. Realiza anotaciones

5. Comenta la lectura con sus compañeros

El primer aspecto se identificó como n/a, es decir no aplica, debido a que el día de la observación, la actividad que realizaron no contenía instrucciones.

En cuanto al aspecto número dos, se concentra al momento de leer, se obtuvo que 26 alumnos sí se concentraron (86\%) y sólo 4 no lograban concentración (14\%) al momento de leer (gráfica 1.11).

\section{Gráfica 1.11}

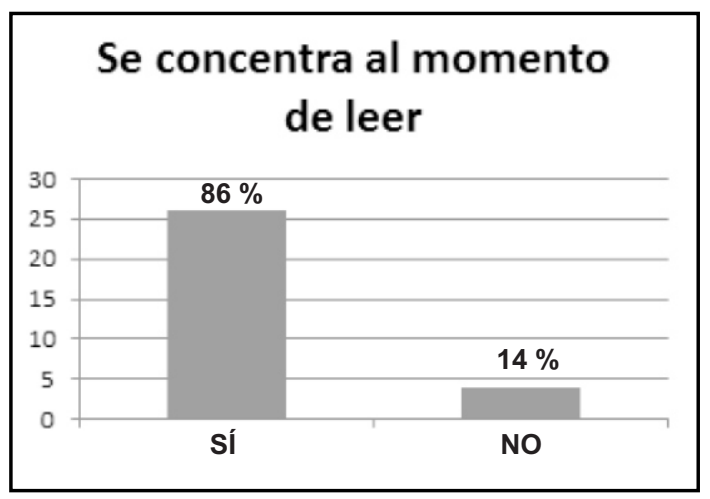

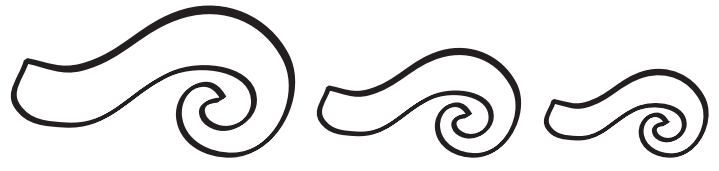

Cuando se observó si los jóvenes subrayaban las ideas principales del texto, se obtuvo que ninguno de los treinta alumnos lo hizo. Un resultado igual se consiguió al observar si realizaban anotaciones sobre el texto que leían.

$\mathrm{Al}$ observar el quinto aspecto que es si el alumno comenta la lectura con sus compañeros, el resultado fue que cinco jóvenes sí comentaron la lectura con sus compañeros (16\%), mientras que los otros 25 alumnos (84\%) no hicieron comentarios (gráfica 1.12).

\section{Gráfica 1.12}

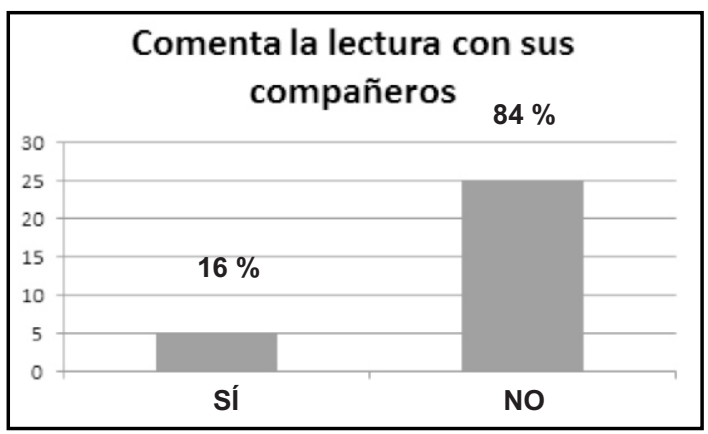

\section{Resultados}

A partir de la información previamente analizada en las encuestas aplicadas a padres de familia y maestros de la comunidad de Ayahualulco, se derivan los siguientes resultados acerca de la comprensión lectora.

Con base en el resultado de preguntas externas a la entrevista aplicada se encontró que el 61\% (19 de 31) de los padres de familia no saben leer, esto dificulta el hábito de la lectura de los hijos.

Por otro lado, González (2007) señala que la comprensión lectora es "una reinterpretación significativa y personal de los símbolos verbales impresos, que se justifica solo en la medida en que el lector es capaz de comprender los significados que están a su disposición. (González, 2007). Los resultados señalan que los padres de familia pueden notar que sus hijos 


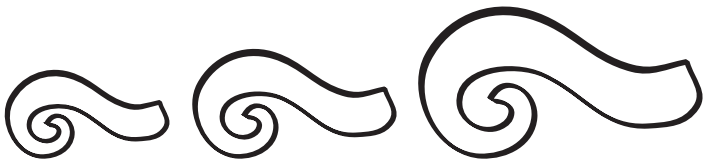

están comprendiendo la lectura y les deja un aprendizaje significativo ya que el 90\% (28 de 31) de los padres encuestados respondió que sus hijos comprenden la lectura y ellos lo notan ya que realizan diferentes acciones para saberlo como preguntas abiertas, resúmenes o platican la lectura, también el 80\% (25 de 31) de los padres respondió que es una acción que les permite adquirir un aprendizaje significativo que pueden llevar a la práctica.

Asimismo Colomer (1993) señala que enseñar a entender un texto, se ha convertido en el objetivo principal en las prácticas escolares y esto ha permitido experimentar y articular con nuevas prácticas para obtenerlo. Esto hace referencia a que un porcentaje de $88 \%$ (8 de 9) de los maestros encuestados de la comunidad de Ayahualulco dedican más de 20 minutos a la lectura con sus alumnos.

Con base en la revisión de las treinta historias de vida aplicadas a alumnos de 5to semestre de bachillerato, se encontró que el $86 \%$ de los alumnos (es decir 26) tienen faltas de ortografía. La Secretaría de Educación Pública (s/f), escribe que la lectura es importante para aumentar el vocabulario y mejorar la ortografía, en este caso podría decirse que no se le ha dado la importancia a la ortografía dentro del salón de clases.

Al momento de la aplicación del instrumento de observación, no se pudo comprobar si los alumnos leen las instrucciones antes de realizar una actividad; sin embargo, al analizar las historias de vida, se pudo constatar que el $16 \%$ de los treinta alumnos no leyeron las instrucciones contenidas en la historia de vida y por ende no relataron lo que se les pidió.

De las treinta historias de vida, se anularon dos por omisión; de las veintiocho válidas se encontró que el $57 \%$ de los alumnos lograron extraer el significado de la lectura en el último libro que leyeron. Según Colomer (1993), enseñar a entender un texto se ha convertido en el

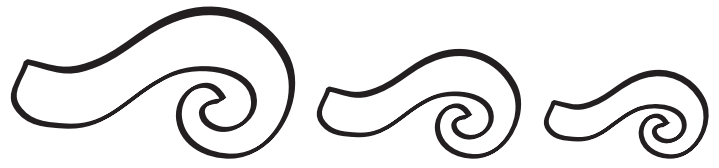

objetivo principal en las prácticas escolares; se puede decir que el otro $43 \%$ de los alumnos no han aprendido a obtener el significado de un texto.

El 46\% de los jóvenes, leyeron a solas su último libro, argumentado que así se logra mayor concentración, según la SEP (s/f) la lectura potencia la capacidad de observación, de atención y de concentración.

\section{Conclusiones}

- Los habitantes de la comunidad de Ayahualulco presentan un problema de lectoescritura, algunos factores que inciden son las condiciones de vida en las que se encuentran, alimentación, tener un lugar en donde vivir y qué vestir es más importante y en resumen, hay pobreza en la comunidad.

- Los habitantes de Ayahualulco consideran que la lectura es importante para aprender y conocer el medio en el que viven, y para la resolución de problemas de la vida cotidiana.

- Dentro de la comunidad de Ayahualulco y sus alrededores, los maestros fomentan la lectura ya que dedican más de 30 minutos en clase para leer.

- Los alumnos del Bachillerato La Salle demostraron tener capacidad de concentración al momento de la lectura, y algunos de ellos mostraron gran capacidad de reflexión.

- A pesar del gran interés por la lectura, los alumnos del Bachillerato La Salle Ayahualulco presentan problemas de ortografía.

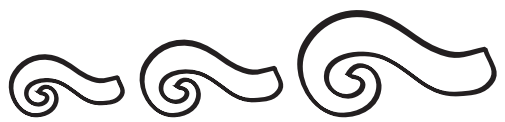




\section{Fuentes de consulta:}

AULA Santillana (2003). Diccionario de las Ciencias de la Educación. México D.F.: Santillana.

COLOMER, T. (1993). La enseñanza y el aprendizaje de la comprensión lectora. Consultado en septiembre 4, 2012 en http://palabrasmaspalabras.bligoo.cl/media/users/14/736597/ files/112715/Comprension.pdf

FERREIRO, E. (2002). Los hijos del analfabetismo: propuesta para la alfabetización en América Latina. México D.F.: Siglo XXI.

FUENTES, L. (2009) Diagnóstico de comprensión lectora en educación básica en Villarrica y Loncoche, Chile Consultado en Septiembre 4, 2012 en: http://www.scielo.org.mx/scielo.php? pid $=$ s0185-26982009000300003\&script $=$ sci_arttext

GONZÁLEZ, A. (2007). ¿Qué se entiende por comprensión lectora? Consultado en septiembre 4, 2012 en http://pedablogia.wordpress.com/2007/05/20/.

IRWIN, J. (1986): Teaching Reading ComprehensionProcesses. Englewood, N.J. PrentinceHall.

MEXICANOS Primero (2011). Nota Técnica. Consultado en septiembre 9, 2012 en http://depanzazo.mx/pdf/Datos-y-afirmaciones-en-De-Panzazo.pdf

SECRETARÍA de Educación de Jalisco (s/f). PRONALEES Programa Nacional para el Fortalecimiento de la Lectura y la Escritura en Educación Básica Consultado en septiembre 4, 2012 en http://portalsej.jalisco.gob.mx/sites/portalsej.jalisco.gob.mx.comunicacionsocial/files/pdf/pronalees.pdf.

SECRETARÍA de Educación Pública (2012). Programa Nacional de Lectura. Consultado en septiembre 9, 2012 en http://lectura.dgme.sep.gob.mx/pnl_dp_00.php.

SECRETARÍA de Educación Pública (s/f). Manual de procedimientos para el fomento y la valoración de la competencia lectora en el aula. Consultado en noviembre 16, 2012 en http://www.dgep.sep.gob.mx/lnKDgep/MANUAL_FOMENTO.pdf

VELÁSQUEZ, Ma. E. (2006), "El nivel de comprensión lectora en sexto grado de primaria” Consultado en Octubre 14, $2012 \mathrm{en:} \mathrm{http://biblioteca.ajusco.upn.mx/pdf/24128.pdf}$

VERACRUZ.com (s/f). Ayahualulco. Consultado en noviembre 8, 2012 en http://veracruz.com/ciudades/ayahualulco
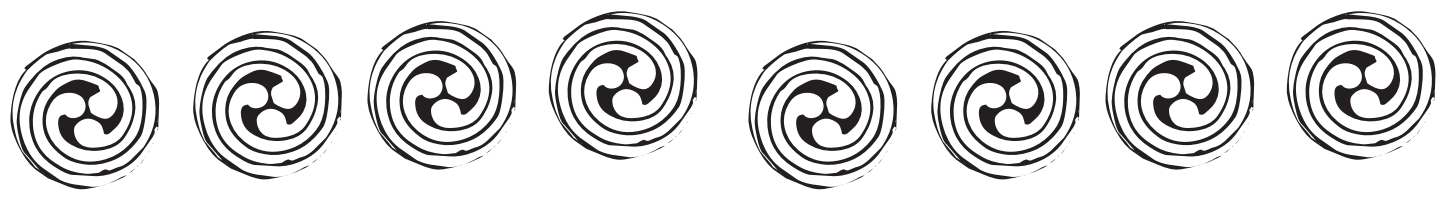

๑5 56 